\title{
Factors affecting the incidence of depression in obese persons
}

\begin{abstract}
Introduction: Many researchers have highlighted a reciprocal link between depression and obesity. This study was an attempt to analyze the effect of various lifestyle factors on the degree of depression assessed using Beck's inventory.

Objectives: To analyse the incidence of depression in obese individuals using a standardised tool named Beck's depression inventory and to analyse the correlation of different lifestyle and dietary factors with depression.

Methodology: The study was conducted in two health care centres in Thiruvananthapuram, Kerala. This experiment was part of a research on obesity and was done among respondents having higher grades of BMI who attempted either bariatric surgery or conventional weight loss methods to reduce weight. The criterion for inclusion in this experiment were BMI of $33 \mathrm{Kg} / \mathrm{m} 2-50 \mathrm{Kg} / \mathrm{m} 2$ and age range $18-65$ years. Those having BMI more than $50 \mathrm{Kg} / \mathrm{m} 2$, history of nephropathy, food allergy and uncontrolled diabetes were excluded. The samples included 10 respondents who opted conventional method and 18 respondents who opted Bariatric surgery for weight loss. The base line data were collected through a structured questionnaire and the depression status was assessed using Beck's depression inventory. A comparative analysis was done using percentage distribution, Pearson correlation coefficient, ANOVA, and Paired t test.
\end{abstract}

Results: The major variable for this experiment was the depression score which generated a mean value of $7.4 \pm 3.9$ in the surgery group and $13.7 \pm 8.2$ in the non surgery group. Age, monthly income, sitting time in hours, sleep apnoea score, waist circumference, Family obesity score and morbidity score were influencing depression status. Body Mass Index and body fat percentage were the most correlating anthropometric parameters with the depression score. Sitting time lower than 5 hours and sleep duration greater than 6hours were related to higher depression score whereas sleep apnoea score did not have any linear relation with depression score. Sedentary occupation and sleep initiation time later than 10 'o clock also related to higher score with depression inventory. Respondents who had a history of obesity from childhood had higher incidence of depression.

The diet analysis revealed that higher intake of protein, carbohydrates and fiber produced a negative trend in the case of depression score while higher intake of fat was producing a positive correlation. The intake of Methionine, phenyl alanin, tryptophan and tyrosine were higher than requirement and had a negative influence on depression. Deficient intake of omega three and omega six fatty acids and excess intake of PUFA and MUFA related to higher scores in depression while adequate or higher intake of EPA and DHA related to lower scores of depression. When the intake of anti oxidant water soluble vitamins were low, the scores obtained with the depression inventory were high. The intake of calcium was close to requirement and was showing somewhat positive influence on depression score. The intake of other important minerals and trace elements were lesser than requirement and had negative influence on depression status. The mean intake of sodium was found to be higher than the recommended intake and produced proportional rise in the depression score.

Summary and conclusion: It is clear from this study that unhealthy lifestyle patterns and poor diet quality have a strong influence on depression score obtained through Beck's inventory.

Keywords: obesity, depression, bariatric surgery, beck's inventory, diet quality
Volume II Issue 3 - 202 I

\section{Krishna Mohandas,' Prema L, ${ }^{2}$ Krishna \\ Kumar S, $^{3}$ Haridas KP ${ }^{4}$}

'Research Scholar in Home Science (Food and Nutrition), Kerala University, India

${ }^{2}$ Former Professor \& Head, Department of Home Science, College of Agriculture, India

${ }^{3}$ Physician \& Nephrologist, Former Head of Nephrology SUT, Hospitals, India

${ }^{4}$ General Gastrointestinal and Laparoscopic surgeon, Chairman and Managing Director, Lords Hospital, India

Correspondence: Krishna Mohandas, Research Scholar, Kerala University, Thiruvanathapuram , Kerala, India, Tel 854737265I, Emailkrishnamohandas@yahoo.co.in

Received:June 10,2021 | Published: June 23, 2021

\section{Introduction}

A person who is obese often faces social stigmatisation in the society and even within families. Social issues and health problems could affect their life to such an extent that they may develop depression. A systematic review and meta analysis of longitudinal studies relating to overweight, obesity and depression by Luppino et al., ${ }^{1}$ confirmed a reciprocal link between depression and obesity. This study was an attempt to analyze the effect of various lifestyle factors on the degree of depression assessed using Beck's inventory which is a standardised questionnaire to score depression.

\section{Objectives}

To analyse the incidence of depression in obese individuals using a standardised tool named Beck's depression inventory. To analyse the correlation of different lifestyle and dietary factors with depression. 


\section{Methodology}

This experiment was part of a research on obesity and was done among respondents having higher grades of BMI who attempted either bariatric surgery or conventional weight loss methods to reduce weight. The study was conducted in one bariatric centre and one general clinic in Thiruvananthapuram, Kerala. Back ground details of the respondents were collected using a structured questionnaire. The variables elicited include demographic data, activity pattern, occupation data, anthropometric, biochemical, clinical and dietary data. Detailed food frequency and recall data was collected through this schedule to derive individual nutrient intake and diet quality. The depression level was assessed using a standard tool named Beck's inventory. The depression inventory contained 21 statements which were converted into local language so study participants could fill them on their own. A score below10 is considered normal, 11 to16 as mild disturbance and above 16 as depression of various degree. All the respondents were enrolled to the study in the first visit when the baseline data were collected. Depression level was tested on first follow up. The study period was from 2015-2017 for the bariatric surgery group and the comparative study from 2016 to 2019 .

\section{Inclusion and exclusion criteria}

Respondents from both the genders in the age range of 18 65 years, with BMI greater than $33 \mathrm{Kg} / \mathrm{m}^{2}$ and less than $50 \mathrm{Kg} / \mathrm{m}^{2}$ were included in the study. The respondents having BMI more than $50 \mathrm{Kg} / \mathrm{m}^{2}$ and those with history of nephropathy, food allergy and uncontrolled diabetes were excluded.

\section{Sample size}

Final sample included 18 respondents who underwent bariatric surgery and 10 respondents who opted diet and exercise to lose weight. A comparative analysis was done using percentage distribution, Pearson correlation coefficient, ANOVA, and Paired t test were the tools utilised in statistical analysis of the data.

\section{Tools used}

A structured questionnaire for collecting demographic, anthropometric, biochemical, clinical and dietary history of respondents, Alternate Healthy Eating Index (AHEI) to assess diet quality and Beck's Depression Inventory were the tools used in the study.

\section{Results}

The basic demographic parameters of both the groups were subjected to comparative analysis. In both the groups, females were more. The mean age in both the groups $(39.4 \pm 8.7$ years in surgery group and $36.3 \pm 9.4$ years in the comparison group) indicated that majority of the respondents were in their prime youth. The income level of the respondents in the surgery group was $22692 \pm 20279$ rupees per month and was $7350 \pm 6439$ rupees in the second group. The education level, occupation, activity level, affluence level and exercise pattern were coded and generated a lifestyle score for ease of analysis. This score was also not much different among the groups (18.05 \pm 1.69 in surgery group and $17.7 \pm 1.49$ in the non-surgery group).

In both the groups, all the parameters which are used to interpret degree of obesity through anthropometric data placed the respondents in the category of grade II obesity and higher grades.

The visceral fat percentage and waist circumferences were far higher than the normal values. The biochemical profile were analysed and all the respondents were found to have either normal haemoglobin level or mild anaemia, dyslipidemia expressed mainly as deficient HDL, normal hepatic profile, and either normal or mildly elevated uric acid level. Albuminurea was more prevalent than glycosurea, and other parameters provided altered results when there were associated medical illnesses. The clinical signs and symptoms associated with obesity were analysed and produced a mixed picture. Rather than obesity per se, higher morbidity levels exhibited corresponding lower quality in clinical signs.

Similarly dietary intake was widely varying. The quality of diet was analysed using Alternate healthy eating index which indicated a better diet quality when the score was high. The average intake of important nutrients and the AHEI scores obtained are presented in the following table. It can be seen that both the groups did not differ much among themselves in the tested parameters. The surgery group had a notably high intake in the case of proteins while the non surgery group had high intake for fat from diet (Tables $1 \& 2$ ).

Table I Comparison of anthropometric data

\begin{tabular}{lll}
\hline Parameters & Surgery group & Non surgery group \\
\hline & $(\mathrm{n}=18)$ & $(\mathrm{n}=10)$ \\
& Mean \pm SD & Mean \pm SD \\
Weight in Kg & $108.06 \pm 15.83$ & $98.6 \pm 21.46$ \\
BMl in Kg/m & $40.93 \pm 4.12$ & $37.23 \pm 3.52$ \\
Body fat percentage & $41.19 \pm 5.27$ & $40.71 \pm 2.89$ \\
Visceral fat percentage & $24.63 \pm 5.7$ & $21.75 \pm 5.09$ \\
Neck circumference $(\mathrm{cm})$ & $40.47 \pm 3.95$ & $38.2 \pm 3.16$ \\
Waist circumference $(\mathrm{cm})$ & $120.72 \pm 10.97$ & $111.1 \pm 14.92$ \\
Waist to Hip ratio & $0.93 \pm 0.08$ & $0.91 \pm 0.14$ \\
\hline
\end{tabular}

Table 2 Comparison of diet quality and macro nutrient intake of surgery and non surgery groups

\begin{tabular}{lll}
\hline Parameter & Surgery group & Non surgery group \\
\hline & $(n=18)$ & $(n=10)$ \\
& Mean \pm SD & Mean \pm SD \\
Alternate Healthy Eating & $49.9 \pm 11.1$ & $54.6 \pm 9.8$ \\
$\begin{array}{l}\text { Index (AHEI) } \\
\text { Energy intake in Kcals }\end{array}$ & $1730.64 \pm 827.26$ & $1633.98 \pm 306.76$ \\
Protein in gram & $69.42 \pm 32.63$ & $61.51 \pm 17.57$ \\
Fat in gram & $39.83 \pm 15.99$ & $51.13 \pm 31.82$ \\
Carbohydrates in gram & $276.08 \pm 158.73$ & $238.15 \pm 76.84$ \\
\hline
\end{tabular}

The major variable for this experiment was the depression score which generated a mean value of $7.4 \pm 3.9$ in the surgery group and $13.7 \pm 8.2$ in the non surgery group. The non surgery group had higher score indicating that they were suffering from depression more than the respondents who opted surgery. To test whether the differences among both the groups were significant, $\mathrm{t}$ test was carried out and the following result was obtained (Table 3 ). 
Table 3 Between group comparison of surgery and non surgery groups

\begin{tabular}{llll}
\hline Variable & Surgery group $(\mathbf{n}=\mathbf{1 8})$ & Non surgery group & P value \\
\hline & $($ Mean \pm SD $)$ & $(\mathrm{n}=10)$ & \\
& & $($ Mean \pm SD $)$ & \\
AHEI & $49.9 \pm 11.1$ & $54.6 \pm 9.8$ & 0.2957 \\
BMI & $40.93 \pm 4.12$ & $37.23 \pm 3.52$ & 0.0295 \\
Lifestyle score & $18.05 \pm 1.69$ & $17.7 \pm 1.49$ & 0.5848 \\
Depression score & $7.4 \pm 3.9$ & $13.7 \pm 8.2$ & 0.0144 \\
Energy intake & $1730.64 \pm 827.26$ & $1633.98 \pm 306.76$ & 0.7342 \\
Protein intake & $69.42 \pm 32.63$ & $61.51 \pm 17.57$ & 0.4988 \\
Fat intake & $39.83 \pm 15.99$ & $51.13 \pm 31.82$ & 0.2396 \\
\hline
\end{tabular}

Paired $t$ test was conducted to analyse the between group difference with a $5 \%$ significance level with a $\mathrm{p}$ value of $<0.05$ indicating a statistical significance and it can be concluded from this table that the differences were not statistically significant though the BMI and depression score were differing among them.
A detailed analysis was done to test the influence of different lifestyle and nutritional parameters on the depression score of the all 28 respondents. Pearson correlation analysis was done with a negative value indicating negative correlation and positive value indicating positive correlation with depression score. The following Tables 4 \& 5 discuss the findings of the analyses.

Table 4 Correlation of lifestyle factors and anthropometric data with depression

\begin{tabular}{ll}
\hline Variable & $\begin{array}{l}\text { Correlation coefficient } \\
\text { with depression }\end{array}$ \\
\hline Age & -0.092 \\
Monthly income & -0.0658 \\
Lifestyle score & 0.0392 \\
Sitting time & -0.1469 \\
Total sleep duration & 0.0794 \\
Sleep apnoea score & -0.0591 \\
Family obesity score & 0.321 \\
Morbidity score & 0.0598 \\
BMl & 0.0498 \\
Fat percentage & 0.1213 \\
Waist circumference & -0.1709 \\
\hline
\end{tabular}

Table 5 Correlation of macronutrient and specific amino acid intakes with depression

\begin{tabular}{llll}
\hline Nutrients & RDA for adults & Mean intake & $\begin{array}{l}\text { Correlation coefficient } \\
\text { with depression }\end{array}$ \\
\hline Protein $[\mathrm{gm}]$ & $58.16 \pm 8.35$ & $66.6 \pm 28.4$ & -0.1548 \\
Total Fat $[\mathrm{gm}]$ & $24.23 \pm 3.42$ & $43.9 \pm 23.6$ & 0.2452 \\
Total Dietary Fibre $[\mathrm{gm}]$ & 35 & $29.2 \pm 20.7$ & -0.0519 \\
Carbohydrate $[\mathrm{gm}]$ & $250.86 \pm 36.17$ & $262.5 \pm 136.5$ & -0.0637 \\
Energy in Kcal $[\mathrm{Kcal}]$ & $1454.29 \pm 209.27$ & $1696.1 \pm 689.7$ & -0.0132 \\
Methionine $[\mathrm{mg}]$ & $15 \mathrm{mg} / \mathrm{Kg}$ & $1565.4 \pm 860.4$ & -0.0381 \\
& $(900 \mathrm{mg}$ for a $60 \mathrm{Kg}$ man $)$ &
\end{tabular}


Table Continued..

\begin{tabular}{llcl}
\hline Nutrients & \multicolumn{1}{l}{ RDA for adults } & Mean intake & \multicolumn{1}{l}{$\begin{array}{l}\text { Correlation coefficient } \\
\text { with depression }\end{array}$} \\
\hline Phenylalanine $[\mathrm{mg}]$ & $30-40 \mathrm{mg} / \mathrm{kg}$ & $3157.5 \pm 1559.1$ & -0.114 \\
& $(1800-2400 \mathrm{mg}$ for a $60 \mathrm{Kg} \mathrm{man})$ & \\
Tryptophan $[\mathrm{mg}]$ & $360 \mathrm{mg}$ & $622.6 \pm 313.8$ & -0.0985 \\
Tyrosine $[\mathrm{mg}]$ & $7 \mathrm{mg} / \mathrm{Kg}$ & $2303.1 \pm 1174.3$ & -0.1296 \\
& $(420 \mathrm{mg} /$ day for a $60 \mathrm{~kg}$ man $)$ & \\
\end{tabular}

The results of this analysis indicate that age, monthly income, sitting time in hours, sleep apnoea score, and waist circumference were negatively influencing depression status. An increase in these parameters were producing a decrease in depression score, while the other parameters tested produced a positive influence on depression status. Lifestyle score included activity level, education, occupation and affluence score, which when increased, showed higher scores with depression inventory. As the sleep duration increased, the score also increased which could be due to the disruptions in the circadian rhythm. Researchers from Final and in $2012^{2}$ has indicated that diet quality can influence sleep quality and duration. They have pointed out that diet rich in fruits and vegetables, low fat proteins, rich in tryptophan (precursor of serotonin, neurotransmitter that induces calmness), B group vitamins and minerals especially magnesium can improve sleep. Family obesity score and morbidity score also influenced depression in positive direction which might be due to the psychological stress associated with higher incidence of obesity in the family and the health issues faced by respondents when the occurrence of co morbid conditions were high. Body Mass Index and body fat percentage were the most correlating anthropometric parameters with the depression score. When the relationship between mental illness, general medical co morbidity and premature mortality were studied by Druss etal., ${ }^{3}$ four modifiable risk behaviours were identified namely tobacco use, substance use, poor diet and lack of physical activity. From the table, it can be concluded that middle age respondents had higher depression scores, lower per capita income and higher lifestyle score related to high depression score. Sitting time lower than 5 hours and sleep duration greater than 6hours were related to higher depression score whereas sleep apnoea score did not have any linear relation with depression score.

A 2014 study from Australia4 established a U shaped relation between Depression and body weight with higher prevalence among underweight and obese general population. ${ }^{4}$ An Iranian study conducted by Tashkori et al., 5 among adolescent girls revealed that the relationship between obesity and depression was strong and positive. A similar Indian study by Neelam Rathee ${ }^{6}$ using Becks inventory in 17-20 year aged females also obtained a strong positive correlation between the level of obesity severity of depression (Tables $6 \& 7$ ).

Table 6 Influence of major fatty acids on depression score

\begin{tabular}{llll}
\hline Nutrient & RDA for adults & Mean intake & $\begin{array}{l}\text { Correlation coefficient } \\
\text { with depression }\end{array}$ \\
\hline Linoleic acid Cl8:2n6 $[\mathrm{mg}]$ & $2500-9000 \mathrm{mg}$ & $3897.4 \pm 3153.4$ & 0.0568 \\
Linolenic acid Cl8:3n3 [mg] & $>500 \mathrm{mg}$ & $270.9 \pm 144.5$ & 0.0326 \\
Eicosapentaenoic C20:5n3 [mg] & EPA+DHA= & $183.3 \pm 269.9$ & -0.1251 \\
Docosahexaenoic C22:6n3 [mg] & $250 \mathrm{mg} /$ day & $335.6 \pm 434.9$ & -0.2866 \\
Total Monuunsaturated Fatty Acids $[\mathrm{mg}]$ & $15-20 \%$ energy from fat & $6717.6 \pm 7463.8$ & 0.1705 \\
Total Polyunsaturated Fatty Acids $[\mathrm{mg}]$ & $6-10 \%$ energy from fat & $5276.4 \pm 3737.5$ & 0.0258 \\
& $250-2000 \mathrm{mg} /$ day & & \\
\hline
\end{tabular}

Table 7 Influence of micronutrient intake on depression score

\begin{tabular}{llll}
\hline & RDA for adults & Mean intake & $\begin{array}{l}\text { Correlation coefficient } \\
\text { with depression }\end{array}$ \\
\hline Thiamine BI $[\mathrm{mg}]$ & $1.2(\mathrm{men})$ I.0(women) & $0.96 \pm 0.76$ & -0.1218 \\
Riboflavin B2 [mg] & $1.4(\mathrm{men})$ I.I(women) & $0.55 \pm 0.33$ & -0.0323 \\
Total B6 [mg] & 2 & $1.42 \pm 0.69$ & $-0.124 \mathrm{I}$ \\
Total Folates B9 $[\mu \mathrm{g}]$ & 200 & $174.2 \pm 76.9$ & -0.0539 \\
Cholecalciferol D3 $[\mu \mathrm{g}]$ & 10 & $2.07 \pm 3.27$ & 0.1337 \\
Beta Carotene $[\mu \mathrm{g}]$ & 4800 & $853.1 \pm 592.2$ & $-0.045 \mathrm{I}$ \\
Calcium $[\mathrm{mg}]$ & 600 & $448.5 \pm 213.9$ & 0.0191 \\
Chromium $[\mathrm{mg}]$ & 0.05 & $0.05 \pm 0.02$ & -0.0998 \\
\hline
\end{tabular}


Table Continued...

\begin{tabular}{|c|c|c|c|}
\hline & RDA for adults & Mean intake & $\begin{array}{l}\text { Correlation coefficient } \\
\text { with depression }\end{array}$ \\
\hline \multirow[t]{2}{*}{ Iron [mg] } & 17(men) & $10.4 \pm 7.56$ & -0.0756 \\
\hline & 21 (women) & & \\
\hline Lithium [mg] & $14.3 \mathrm{mcg} / \mathrm{Kg}$ (Img for a $70 \mathrm{Kg}$ adult man) & $0.03 \pm 0.11$ & -0.1578 \\
\hline Magnesium [mg] & 310 (women), 340 (men) & $346.5 \pm 234.7$ & -0.1343 \\
\hline \multirow[t]{2}{*}{ Potassium $[\mathrm{mg}]$} & 3750 (men) & $|86| .6 \pm 832 . \mid$ & -0.0523 \\
\hline & 3225 (women) & & \\
\hline Selenium [mcg] & $71-163$ & $100.7 \pm 107.4$ & -0.0429 \\
\hline \multirow[t]{2}{*}{ Zinc $[\mathrm{mg}]$} & 12 (men) & $7.18 \pm 5.29$ & -0.0588 \\
\hline & 10 (women) & & \\
\hline Total polyphenols $[\mathrm{mg}]$ & DRI not fixed & $135.9 \pm 68.7$ & 0.0569 \\
\hline \multirow[t]{2}{*}{ Total sodium $[\mathrm{mg}]$} & 2100 (men) & $3882.9 \pm 1137.3$ & 0.2111 \\
\hline & 1900 (women) & & \\
\hline lodine $[\mu \mathrm{g}]$ & 150 & $123.3 \pm 36.7$ & -0.0584 \\
\hline
\end{tabular}

In 2015, Rosengren et al., ${ }^{7}$ conducted a 17 country wide cross sectional study investigated the association between psychosocial factors and general and abdominal obesity and revealed that individuals with permanent stress tended to be slightly more obese but did not support a causal link between psychosocial factors and abdominal obesity. A French study by Chauvet-Gelenier et al., ${ }^{8}$ reviewed the relation between depression and obesity across different geographical areas and confirmed significant bidirectional relationships between depression and obesity at a group level. A 2020 review by Blasco etal., ${ }^{9}$ investigated the researches on the relation between depression and obesity and demonstrated that there is a link between them but the significance of association could not be established.

An Indian cross sectional study by Agarwal et al., ${ }^{10}$ analysed the incidence of metabolic syndrome and central obesity in depression and demonstrated significantly more incidence of metabolic syndrome and central obesity in patients of depression than age and sex matched controls.

Garg et al., ${ }^{11}$ conducted a study of association of obesity with depression in armed forces personnel and revealed that the likelihood of depression was most strongly associated with BMI followed by age, status of living with family and habit of drinking alcohol.

Analysis of variance was done with gender, type of occupation, sleep initiation time, and childhood obesity to test whether these variables were influencing depression. Gender based analysis proved that as the number of female respondents were more, depression scores were higher for females. Sedentary occupation and sleep initiation time later than 10 'o clock also related to higher score with depression inventory. Respondents who had a history of obesity from childhood had higher incidence of depression as adults compared to those who did not have such history. The $p$ values obtained were $0.6501,0.7954$, 0.7954 , and 0.0958 indicating that there was no statistically significant influence but childhood obesity had the strongest influence.

A 2008 study by Simon et al., ${ }^{12}$ analysed the association between obesity and depression in middle aged women and found out that depression is strongly and consistently associated with obesity, lower physical activity, and (among the obese) higher caloric intake. A prospective observational study from Kerala conducted by
Ananthasayanam et al., ${ }^{13}$ revealed that majority of overweight and obese individuals were unaware of the seriousness and health hazards of obesity.

Next tables describe the influence of dietary factors which might influence depression status. On reviewing literature, it was found that higher intake of fruits, vegetables, and low GI carbohydrates are helpful in reducing depression while consumption of high GI carbohydrates and alcohol consumption were detrimental to mental health. ${ }^{14}$ The Alternate Healthy Eating Index is a tool which takes in to account all these parameters and predicts better quality of diet when the score is high. A 2019 study by Parletta et al., ${ }^{15}$ investigated the role of Mediterranian style diet supplemented with fish oil to improve diet quality and mental health in people with depression found that diet high in fruits, vegetables, nuts, legumes, whole grains and omega 3 fatty acids are protective against depression while unhealthy snacks, red meat, chicken, processed foods, high fat high sugar foods, and deficit of omega six fatty acids had an opposite effect. A systematic review by O'Neil et al., ${ }^{16}$ highlighted similar findings in the case of children and adolescents. ${ }^{16}$

Researchers in the field of depression has highlighted the relationship of specific nutrients on the level of depression. Nutrients which are found to be protective in depressive disorders are omega three fatty acids, folate, zinc, magnesium, amino acids tryptophan, tyrosine, Phenyl alanine and Methionine, vitamin B12, B1, B2,B6, iron, calcium, chromium, iodine, lithium, zinc and selenium. Nutrients identified to have negative impact are trans fats, high GI carbohydrates, saturated fatty acids, protein deficiency, very low carbohydrate content in diet and refined carbohydrates. ${ }^{17}$ Alternate healthy eating index is a tool used to interpret the quality of diet in terms of inclusion of whole grain cereals, vegetables, fruits, omega 3 PUFAs, sugar sweetened beverages, salt intake and alcohol consumption. ${ }^{18}$ The vitamin, mineral, and essential fatty acid requirement were derived from the nutrient requirement and recommended dietary allowances for Indians published by ICMR in $2010 .{ }^{19}$ The requirement of specific amino acids, Methionine, ${ }^{20}$ phenyl alanine, ${ }^{21}$ tryptophan, and tyrosine ${ }^{22}$ were obtained from various Indian and international research studies. The references for lithium intake was obtained from a 2018 study by Szklarska and Rzymski ${ }^{23}$ and data 
on polyphenols from study conducted by Cori, et al24. As stated, the overall quality of diet was analysed using AHEI and the correlation with depression produced a negative influence indicating that as the diet quality reduces, depression increases ( $p$ value -0.264 ). The effect of individual nutrients on depression was analysed in detail.

The macronutrient requirements were calculated based on adjusted ideal weight and used for comparison with actual intake. ${ }^{24}$ The analysis revealed that higher intake of protein, carbohydrates and fiber produced a negative trend in the case of depression score while higher intake of fat was producing a positive correlation. This observation was similar to the available research findings. In this study finding, it can be seen that the intake of Methionine, phenyl alanin, tryptophan and tyrosine intake were higher than requirement and had a negative influence on depression indicating that adequate or excess intake of these amino acids are protective against depression.

From the table it was clear that the intake of omega three and omega six fatty acids were lesser than requirement and had a positive correlation. The intake of fish was generally high among the respondents. The combined intake of Eicosa pentanoic acid and Docosa hexanoic acid was higher than the requirement and the influence on depression was negative. The intake of PUFA and MUFA were higher than requirement and had a positive influence on depression. Thus it can be concluded that deficient intake of omega three and omega six fatty acids and excess intake of PUFA and MUFA related to higher scores in depression while adequate or higher intake of EPA and DHA related to lower scores of depression.

The analysis of vitamin intake revealed that the intake of thiamine, riboflavin, B6, folates, and beta carotene were lesser than the requirement and had a negative influence on depression score. The intake of Cholecalciferol was also less but exhibited opposite trend in depression status. Polypenol intake also had a positive correlation. Thus it was clear that when the intake of anti oxidant water soluble vitamins were low, the scores obtained with the depression inventory were high.

The intake of calcium was close to requirement and was showing somewhat positive influence on depression score. The intake of other important minerals and trace elements viz. chromium, iron, lithium, magnesium, potassium, selenium, zinc and iodine were lesser than requirement and had negative influence on depression status. In line with the available research findings, this study also revealed that deficiency of these minerals related to higher scores in depression. The mean intake of sodium was found to be higher than the recommended intake and produced proportional rise in the depression score.

\section{Summary}

Age, Monthly income, sitting time in hours, and waist circumference had a negative influence on depression score. Better lifestyle, longer sleep duration, delayed sleep initiation time, high incidence of obesity in family, high morbidity level, higher Body Mass index and higher body fat percentages had a positive influence on depression level. Similarly incidence of obesity from childhood, sedentary occupation, and poor diet quality had a positive influence on depression. Higher intake of protein, total carbohydrates and fiber was related to lower scores in depression while higher intake of total energy and fat had opposite influence on depression. Lower than required intake of thiamine, riboflavin, pyridoxine, folate, beta carotene, iron, lithium, magnesium, potassium, selenium, zinc, omega 3 and omega 6 fatty acids, essential amino acids like Methionine, phenyl alanine, tryptophan, tyrosine, and iodine had a negative influence on depression level. Excess intake of sodium had a positive influence on depression.

\section{Conclusion}

Thus it is clear from this study that unhealthy lifestyle patterns and poor diet quality have a strong influence on depression score obtained through Beck's inventory.

\section{Acknowledgments}

University Grants Commission.

\section{Conflicts of interest}

None.

\section{Funding}

None.

\section{References}

1. Floriana S Luppino, Leonore $\mathrm{M}$ de Wit, Paul $\mathrm{F}$ Bouvy, et al Overweight,Obesity and Depression- A Systematic Review and Metaanalysis of Longitudinal Studies. Arch Gen Psychiatry. 2010;67(3):220 229.

2. Katri Peuhkuri, Nora Sihvola, Riitta Korpela. Diet promotes sleep duration and quality. Nutr Res. 2012;32(5):309-319.

3. Benjamin G Druss, Lydia Chwastiak 1, John Kern, et al. Psychiatry's Role in Improving the Physical Health of Patients With Serious Mental Illness: A Report From the American Psychiatric Association. Psychiatric Service.s 2018;69(3):254-256.

4. Mariko Carey, Hannah Small, Sze Lin Yoong, et al.Prevalence of Comorbid Depression and obesity in General Practice: A Cross-sectional Survey. British Journal of General Practice. 2014;64(620):e122-e127.

5. Tashkori A, Riahi F, Mohammadpour A. The Relationship between Body Mass Index and Depression among High School Girls in Ahvaz. Hindawi Publishing Corporation: Advances in Medicine. 2016;3645493:5.

6. Neelam Rathee. Obesity and Depression: Are they related? Indian Journal of Health and Well being. 2017;8(12):1472-1475.

7. A Rosengren, $\mathrm{K}$ Teo, S Rangarajan, et al. Psychosocial factors and obesity in 17 high-, middle- and low-income countries: the Prospective Urban Rural Epidemiologic study. International Journal of Obesity 2015:39:1217-1223.

8. Chauvet-Gelinier JC, Roussot A, Cottenet J, et al. Depression and obesity, data from a national administrative database study: Geographic evidence for an epidemiological overlap. PLoS ONE. 2019;14(1): e0210507.

9. Blasco BV, Garcis-Jimenez J, Bodoano I, et al. Obesity and Depression: Its Prevalence and Influence as a Prognostic Factor: A Systematic Review. Psychiatry Investig. 2020;17(8):715-724.

10. Agarwal A, Agarwal M, Garg K, Dalal, et al. Metabolic syndrome and central obesity in depression: A cross-sectional study. Indian J Psychiatry. 2016;58:281-6.

11. Garg R, Saxena SK, Bashir S. Is obesity a risk to depression? A crosssectional study. Ind Psychiatry J. 2019;28:130-134.

12. Simon GE, Ludman EJ, Linde JA, et al. Association between Obesity and Depression in Middle-aged Women. Gen Hosp Psychiatry. 2008;30(1): 32-39.

13. Ananthasayanam A, Kannan S, Dinsar M, et al. Prevalence of obesity and its link between various disorders in a semi urban district in Kerala. International Journal of Pharmaceutical Sciences and Research. 2016;7(9):3826-3834 
14. Natalie Parletta, Dorota Zarnowiecki, Jihyun Cho, et al. A Mediterraneanstyle dietary intervention supplemented with fish oil improves diet quality and mental health in people with depression: A randomized controlled trial (HELFIMED). Nutritional Neuroscience. 2019;22(7):474- 487.

15. Adrienne O’Neil, Shae E Quirk, Siobhan Housden, et al. Relationship Between Diet and Mental Health in Children and Adolescents: A Systematic Review). Am J Public Health. 2014;104:e31-e42.

16. Sathyanarayana Rao TS, Asha MR, Ramesh BN, et al. Understanding Nutrition, Depression and mental illness. Indian $J$ Psychiatry. 2008;50(2):77-82.

17. Chiuve SE, Fung TT, Rimm EB, et al. Alternative Dietary Indices Both Strongly Predict Risk of Chronic Disease. J Nutr. 2012;142:1009-1018.

18. Nutrient requirements and Recommended Dietary Allowances for Indians - A report of the expert group of the Indian Council of Medical Research. ICMR. reprint-2013; 2010.

19. Anura V Kurpad, Meredith M Regan, Sureka Varalakshmi, et al. Daily methionine requirements of healthy Indian men, measured by a $24-\mathrm{h}$ indicator amino acid oxidation and balance technique. Am J Clin Nut. 2003;77(5):1198-205.
20. Anura V Kurpad, Meredith M Regan, Tony DS Raj, et al. The daily phenylalanine requirement of healthy Indian adults. Am J Clin Nutr. 2006;83:1331-1336.

21. Susan A Roberts, Jane M Thorpe, Ronald O Ball, et al. Tyrosine requirement of healthy men receiving a fixed phenylalanine intake determined by using indicator amino acid oxidation. Am J Clin Nutr. 2001;73:276-282.

22. Szklarska D, Rzymski P. Is Lithium a Micronutrient? From Biological Activity and Epidemiological Observation to Food Fortification. Biological Trace Element Research. 2019;189:18-27.

23. Cory H, Passarelli S, Szeto J, et al. The Role of Polyphenols in Human Health and Food Systems: A Mini-Review. Front Nutr. 2018;5:87.

24. Clinical Dietetics Mannual. Indian Dietetic Association. $1^{\text {st }}$ ed. 2011. 10 p. 